\title{
Los arreglos espaciales en la producción comercial del maíz tropical
}

\author{
Spatial arrangements in the commercial tropical maize productión
}

${ }^{1}$ Pedro García Mendoza ${ }^{a}$

\section{RESUMEN}

El cultivo de maíz en el Perú es considerado el segundo rubro de mayor importancia económica y social en el país, después de la papa, teniendo un alto valor estratégico, debido a su distribución geográfica y al papel que desempeña en la alimentación humana y animal. A pesar de la relevancia de este cultivo para la población peruana, existen aún muchos factores de tipo agronómico que están restringiendo los niveles de productividad del cultivo, los cuales se acentúan en la región de la sierra peruana. El principal objetivo de este trabajo fue analizar el efecto que tienen los arreglos espaciales en maíz sobre la producción de grano y biomasa en general, como uno de los factores que tienen mayor influencia en la producción de maíz comercial para grano o forraje en las regiones tropicales y muy particularmente, en la región de la sierra peruana. El análisis se efectuó en función de los resultados de investigaciones desarrolladas en diversos ambientes tropicales del mundo, donde se trató de determinar la influencia que tienen las densidades de población sobre ciertos parámetros del cultivo, que tienen una relación directa sobre la eficiencia de la planta para transformar la energía lumínica en materia seca, tales como el índice de área foliar. También se dan algunas recomendaciones que deben ser considerada al momento de abordar trabajos de investigación científica con este tópico del cultivo en la región de la sierra peruana, como una manera de contribuir en estos futuros estudios, brindando orientaciones técnicas para asegurar el logro de resultados de alto impacto para el cultivo en esta región del Perú.

\begin{abstract}
After the potato, maize is considered the second greater economic and social importance crop in Peru, having a high strategic value, due to its geographical distribution and the role it plays in human and animal food. Despite this great relevance of this crop for the Peruvian population, still there are many agronomic factors that restricting the levels of productivity of the crop, which are felt most acutely in the Peruvian highlands region. In this way, the main objective of this work was to analyze the effect of spatial arrangements on grain and biomass corn production, being one of the factors that have greater influence in the commercial corn production for grain or forage in the tropical regions and more specifically in the Peruvian highlands region. The analysis was showed based on the results of research carried out in different world's tropical environments, where it tried to determine the influence of plant densities on certain crop parameters, which have a direct relationship on the efficiency of the plant to transform the light energy into dry matter, such as the leaf area index. In this paper also we gives some recommendations that should be considered when addressing scientific research work with this maize issue in the Peruvian highlands region, in order to contribute in these future studies, providing technical guidance to ensure the achievement of high impact results for the crop in this region of the country.
\end{abstract}

'Universidad Nacional Autónoma de Tayacaja “Daniel Hernández Morillo” - Huancavelica, Perú

${ }^{a}$ Ing. Agrónomo 


\section{INTRODUCCIÓN}

El cultivo de maíz es una de las fuentes de alimentos y de forraje más importantes del mundo. Dentro de los cereales, representa a la especie con el mayor potencial para el rendimiento de grano y para explorar todo ese potencial que posee la planta para transformar la energía solar en producción de grano, es necesario entender como la planta interactúa morfológica y fisiológicamente en una comunidad de plantas, para identificar o desarrollar prácticas de manejo que permitan maximizar los recursos utilizados en su crecimiento en el ambiente donde se desarrolle el cultivo (Paliwalt Sprague, 1981).

Aunque es uno de los cultivos que se siembra en mayor diversidad de ambientes, desde los $58^{\circ} \mathrm{de}$ latitud norte, en Canadá y Rusia, hasta los $40^{\circ}$ de latitud sur y desde altitudes por debajo del nivel del mar en las planicies del Caspio, hasta por encima de los $3800 \mathrm{msnm}$ en la cordillera de los Andes (Paliwal, 2001), los mayores niveles de productividad se obtienen en los países templados y desarrollados, siendo USA el principal país productor de maíz en el mundo y quien ostenta los niveles más altos de productividad promedio (FAO, 2017).

Las razones por las cuales los maíces en las zonas templadas registran mayores niveles de productividad, comparados con los rendimientos observados en las regiones tropicales, son variadas, pues además de las diferencias en condiciones ambientales, en la mayoría de los países de las regiones templadas el maíz normalmente es cultivado con elevados niveles de manejo agrotécnico, lo que hoy se conoce como el uso de tecnologías de precisión, al contrario de lo que sucede en la mayor parte de los países tropicales, donde el cultivo se produce bajo condiciones de tempero, con elevadas temperaturas diurnas y nocturnas (baja amplitud térmica), con variadas condiciones de humedad, normalmente sujeto a períodos erráticos de estrés por sequía y/o excesos de agua en diferentes etapas de desarrollo del cultivo, con inefectivos controles de las poblaciones de malezas y de los insectos plagas y bajos niveles de fertilización, vale decir, como un cultivo de subsistencia, con muy bajos niveles de manejo y uso deficiente de insumos agrícolas (Paliwal y Sprague, 1981).

No obstante, en la mayoría de los países tropicales los niveles de productividad mejoran significativamente cuando se eleva el uso de niveles tecnológicos (Paliwal y Sprague, 1981). Esto nos sugiere, que a pesar de que existen otros factores, además del manejo agronómico y poco uso de insumos agrícolas, que contribuyen a los bajos rendimientos del maíz tropical, el mejoramiento del manejo agroecológico del cultivo es determinante para incrementar la productividad del maíz en los países tropicales. En el caso particular del Perú, la producción maicera de la región de la sierra está caracterizada como unidades de producción minifundistas, realizada por productores de bajos niveles de instrucción, escasa capacidad empresarial, que practican una actividad agrícola de subsistencia con muy bajos niveles de insumos, a pesar de ser una actividad económica que representa la principal fuente de ingresos para el sustento de la inmensa cantidad de familias que explotan este rubro en la sierra peruana MINAGRI (2012).

Todos estos factores restringen la producción, al punto de que los niveles de productividad de esta región se ubiquen alrededor de $1,8 \mathrm{t} / \mathrm{ha}$, respecto al promedio nacional, estimada en unas 3,3 t/ha (MINAGRI, 2012; FAO, 2017). Sin embargo, diversos trabajos realizados en el cultivo en las regiones tropicales del mundo, demuestras que la densidad de plantas es una de las prácticas culturales de mayor importancia, que determinan 
no sólo el rendimiento de grano, sino además, otros atributos agronómicos importantes del cultivo (Reta et al., 2000; Sangoi, 2000; Cano et al., 2001; Tinoco, 2006; Tinoco et al., 2008; Abuzar et al., 2011; Sangoi et al., 2012.

En este sentido, el principal objetivo de este trabajo es analizar el efecto que tienen los arreglos espaciales en maíz sobre la producción del cultivo, como uno de los factores que tienen mayor influencia en la producción de maíz comercial para grano o forraje en las regiones tropicales y muy particularmente en la región de la sierra peruana.

Densidades de poblaciones en el cultivo del maíz y densidad óptima

A pesar de ser uno de los factores agronómicos que más influencia la producción de maíz en los trópicos (Cervantes et al, 2013), las poblaciones de plantas establecidas en el cultivo de maíz también representan a uno de los aspectos relativamente más fáciles de corregir, cuando se cuenta con la información para tomar decisiones apropiadas. El cultivo del maíz es muy sensible a este factor, pues la planta no es capaz de compensar una baja población y una población por encima de la densidad óptima también resulta altamente perjudicial. En este sentido, Cabrera et al. (2000), demostraron que una planta adicional por metro lineal, por encima de la densidad óptima, podía afectar negativamente la productividad de los híbridos evaluados. Por esta razón, resulta sumamente importante determinar la densidad óptima de producción para cada cultivar desarrollado y para cada localidad donde se piense establecer el cultivo.

Sangoi (2000) y también Cervantes et al. (2013) refieren que cuando el número de plantas por unidad de área supera a la densidad óptima, se generan una serie de consecuencias negativas para el desarrollo de la mazorca que pueden conducir a la esterilidad, incrementándose el número de plantas jorras (plantas sin mazorcas) en las plantaciones comerciales. En primer lugar, la diferenciación de la flor femenina (mazorca) se retarda con relación a la diferenciación de la inflorescencia masculina (panoja) y este retardo en la diferenciación de la flor femenina se traduce en una menor tasa de crecimiento, lo que conlleva a que pocos primordios florales se transformen en espiguillas funcionales durante la floración. Adicionalmente, estas espiguillas funcionales presentan una emisión de estigmas más lenta en comparación a las plantas no estresadas, lo cual incrementa el intervalo de floración masculina y femenina (ASI, por sus siglas en inglés), que disminuye el número de estigmas que pueden ser fertilizados, debido a la falta de sincronía floral. Por último, se producen abortos de los óvulos recién fertilizados, provocados por las limitaciones en la suplencia de carbono y nitrógeno a las mazorca.

La densidad óptima se entiende como la menor densidad que posibilita maximizar el rendimiento en grano, estando la misma en función del ambiente donde el cultivo se desarrolle y del cultivar considerado (Ross, 2012, Videla et al., 2014). Por ello, es un parámetro que debe ser determinado para cada cultivar desarrollado y para cada localidad donde se vayan a utilizar los materiales genéticos. Es importante acotar, que la densidad óptima expresa, de manera indirecta, el mejor arreglo espacial de las plantas en un ambiente determinado, que posibilita el uso más eficiente de la energía solar. De allí que es más recomendable hablar de arreglos espaciales, más que de densidades de población, debido a que dos arreglos espaciales pueden generar una misma densidad de población, pero una de éstas resulta más eficiente en captar la energía lumínica.

De acuerdo a Van Heesmst y Van Keulen (1986) y 
Versteeg y Van Keulen (1986), en las gramíneas pueden distinguirse tres períodos en la tasa de crecimiento; una primera etapa, representada por la fase de crecimiento lento, que comprende la etapa vegetativa entre $\mathrm{V}-1$ y $\mathrm{V}-5$, donde las plantas poseen poco desarrollo, que no se sombrean entre sí y donde la tasa de crecimiento y la acumulación de materia seca se incrementan exponencialmente; una segunda etapa, que corresponde a la fase de crecimiento exponencial, donde el cultivo cubre completamente el suelo, maximizándose la intercepción de luz con una tasa de crecimiento constante, en donde el peso seco se incrementa en forma lineal, y una tercera fase que coincide con la etapa de la senescencia de las hojas, que ocasiona una fuerte caída en la tasa de crecimiento. Sólo la radiación que se encuentra dentro del espectro visible en el rango de los 400 a $700 \mathrm{~nm}$ de longitud de onda, es susceptible de ser usada por el cultivo en el proceso de fotosíntesis e impactar sobre su crecimiento y desarrollo, aunque este rango apenas representa cerca el 0,5 de la radiación global total (Monteith, 1977).

\section{Los arreglos espaciales y el índice de área foliar}

La densidad de población y el arreglo espacial de plantas tienen un efecto directo sobre el crecimiento y desarrollo de las plantas y es de esperar que al variar las distancias entre surcos y entre plantas haya una manifestación diferencial en los valores de índice de área foliar (IAF) y de la capacidad productiva de los genotipos evaluados, todo lo cual se sustenta en los análisis cuantitativos del crecimiento, en donde se requiere medir los materiales considerados y la capacidad de éstos para convertir su estructura vegetal en producción de materia seca total. La forma de conocer a detalle dichos conceptos se logra a través de la cuantificación del peso de materia seca total por unidad de área y la determinación del IAF (Báez et al., 2002). El IAF es una expresión numérica adimensional, que resulta de la división aritmética del área foliar de un cultivo (expresado en m2) y el área de suelo ocupada por el mismo (expresado en m2), tal como se muestra en la ecuación 1. El IAF permite estimar la capacidad fotosintética de las plantas y ayuda a entender la relación entre acumulación de biomasa y rendimiento bajo condiciones ambientales imperantes en una región determinada.

Ecuación 1: IAF $=\frac{(\text { Área Foliar }) x(\text { Densidad de Población })}{\text { Area Sembrada }}$

Se trata de una herramienta útil y precisa para estimar la capacidad de captura de luz del dosel vegetal de un cultivo determinado, el cual está íntimamente ligado a la densidad de población. Aunque la intercepción de luz tiende a saturarse en $\mathrm{IAF}>3$, la distribución de las hojas puede afectar la eficiencia de uso de la luz (De la Casa et al, 2007; Díaz et al, 2008). Por ello es muy importante hablar de arreglos espaciales, más que de densidad de población, porque como ya fue mencionado, el cultivo puede ser establecido con diferentes arreglos espaciales y obtenerse una densidad de población igual o muy similar, pero uno de estos arreglos puede mostrar una mejor distribución espacial de las plantas, que permita un uso más eficiente de la luz interceptada por el cultivo y del espacio físico, lo cual le permite mejores condiciones para competir con otras plantas de su misma especie o de malezas. Por ejemplo, la siembra del cultivo utilizando arreglos espaciales de 0,80 m entre hileras y 5 plantas/m generan una densidad de población de alrededor de 62.500 plantas $/ \mathrm{ha}$; del mismo modo, una siembra efectuada con arreglos espaciales de $0,70 \mathrm{~m}$ y 4,4 plantas $/ \mathrm{m}$, también generan una densidad de población muy similar a la anterior, pero la competencia intraespecífica en la distancia de 0,80 podría ser 
mayor. Todo ello va a depender del cultivar utilizado y del ambiente de evaluación.

Si bien muchos de los estudios efectuados hasta el momento indican una respuesta positiva del cultivo a medida que se disminuyen la distancia entre hileras (Cabrera et al., Reta et al, 2000; Tinoco et al., 2008; Sangoi et al., 2012), en el caso de la sierra peruana, en donde la mayor parte de las labores agronómicas son efectuadas en forma manual, no es práctico reducir las distancias entre hileras por debajo de $0,80 \mathrm{~m}$, debido a que se dificultan las actividades operativas dentro del cultivo. Por esta razón, los estudios que se lleven a cabo para determinar la densidad óptima de los cultivares utilizados por los productores de maíz de esta región, tendrán que tomar muy en cuenta este aspecto antrópico del cultivo, tratando de afinar la cantidad de plantas/m en cada genotipo evaluado y para cada zona en particular, manteniendo la distancia de $0,80 \mathrm{~m}$ entre hileras como un factor fijo.

Uno de los factores que se ha determinado que más afecta la producción del maíz, es la uniformidad en los arreglos espaciales dentro de la hilera, que determina la distancia entre pantas o punto de siembra (este último en las siembras manuales), (Sangoi, 2012). Este es uno de los factores poco considerados por los productores de maíz de la sierra peruana, e incluso por aquellos agricultores con formación profesional en el área agronómica, pues en las siembras manuales se pueden observar puntos de siembras con 4, 5 plantas, e incluso con un mayor número de plantas y otras situaciones en donde la siembra se efectúa a chorro corrido. En ambas situaciones, los efectos son muy negativos para el rendimiento de grano y materia seca total, porque como bien explicaron Cabrera et al. (2000) y Sangoi (2000), el cultivo del maíz es muy sensible a este tipo de situaciones, generándose una alta competencia intraespecífica, que resulta altamente perjudicial para la producción de maíz para grano y forraje.

\section{Consideraciones finales}

La generación de conocimientos que ayuden a explicar la manera en que la densidad de población, el arreglo espacial de las plantas y los propios cultivares utilizados por los productores agrícolas afectan el crecimiento y desarrollo del cultivo y en consecuencia, la producción de grano y de forraje en maíz, implica incursionar en estudios con enfoque de sistemas, en donde una de las herramientas de gran utilidad utilizadas para estudiarlos y analizar su evolución en el tiempo, son los modelos matemáticos (De La Casa et al., 2007; Díaz et al., 2008; Tinoco et al, 2008). Se tratan de una herramienta que posibilita una representación abstracta de la realidad en un lenguaje matemático, los cuales han probado que pueden reproducir el comportamiento de los sistemas como un todo, con un grado aceptable de precisión, al predecir lo que pasaría bajo condiciones diversas, de manera que pueden ser utilizados para tomar decisiones a la hora de introducir cambios en los sistemas de producción agrícola (Quijano et al., 1995, citados por Tinoco et al., 2008). No obstante, a pesar de que las mismas disminuyen los costos de los trabajos de investigación y el tiempo requerido para obtener resultados, requieren ser debidamente validadas en campo, a fin de que puedan generar resultados confiables.

En el Perú y particularmente en la región de la Sierra, son muy pocos los trabajos de investigación que se han realizados para determinar los mejores arreglos espaciales para los diferentes cultivares utilizados por los productores maiceros en la región, que posibiliten el uso más eficiente de la energía lumínica para convertirla en materia seca (Sevilla, 2008; De La Cruz, 2016). 
En este sentido, en la Universidad Nacional Autónoma de Tayacaja "Daniel Hernández Morillo"(UNAT), se están estableciendo diferentes convenios de cooperación interinstitucionales, tanto con los gobiernos locales y regionales, así como también con instituciones nacionales e internacionales, en donde se están abriendo espacios propicios para el desarrollo de proyectos de cooperación de gran relevancia para el cultivo del maíz, con el establecimiento de equipos multidisciplinarios, que puedan abordar temas como este y otros de gran importancia, que sin duda contribuirán a mejorar la productividad del cultivo de maíz en el Perú en el mediano plazo. Para ello también será necesario el uso de semilla de alta calidad, que garantice una adecuada uniformidad en las poblaciones de plantas en las parcelas de los productores agrícolas de esta región peruana.

\section{REFERENCIAS BIBLIOGRÁFICAS}

Abuzar, M. R., Sadozai, G. U., Baloch, M. S., Baloch, A. A., Shah, I. H., Javaid, T. and Hussain, N. 2011. Effect of plant population densities on yield of maize. The Journal of Animal \& Plant Sciences, 21(4): 2011, Page: 692-695.

Báez, G. A. D; Chen, P.; Tiscareño-López, M. and Srinivasan, R. 2002. Using satellite and field data with crop growth modeling to monitor and estimate corn yield in Mexico. Crop Sci. 42(6):1943-1949.

Cabrera, S. R., García, P. J., Barboza, N., Morillo, F., Sánchez, C. 2000. Rendimiento de híbridos de maíz (Zea mays L.) a diferentes arreglos espaciales de siembra en Venezuela. UNELLEZ de Ciencia y Tecno., 18 (1): 142-161.

Cano, O., Hugo Tosquy, O., Sierra, M. y
Rodríguez, F. A. 2001. Fertilización y densidad de población en genotipos de maíz cultivados bajo condiciones de temporal. Agronomía Mesoamericana, 12(2): 199-203.

Cervantes-Ortíz, F., Covarrubias-Prieto, J., Rangel-Lucio, J. A., Terrón-Ibarra, A., Mendoza-Elos, D. M. y Preciado-Ortiz, R. E. 2013. Densidad de población y fertilización nitrogenada en la producción de semilla híbrida de maíz. Agronomía Mesoamericana 24(1):101-110.

De la Casa, A., Ovando, G., Bressanini, L., Rodríguez, Á. y Martínez. J. 2007. Uso del índice de área foliar y del porcentaje de cobertura del suelo para estimar la radiación interceptada en papa. Agricultura Técnica (Chile), 67(1):78-85.

De La Cruz E., J. C. 2016. Fraccionamiento de nitrógeno en dos densidades de siembra de maíz amarillo duro (Zea mays 1.) en la localidad de La Molina. Trabajo de tesis presentado como requisito parcial para optar al título de Ingeniero Agrónomo. Universidad Nacional Agraria La Molina. Facultad de Agronomía. Disponible en: http://repositorio.lamolina.edu.pe/bits tream/ handle/UN A L M / 1961 / F 01 - C 794 T.pdf? sequence $=1 \&$ isAllowed $=\mathrm{y}$.

Díaz, E. A., Acosta Gallegos, J. A., Amador Ramírez, M. D. y Padilla Ramírez, J. S. 2008. Relación entre índice de área foliar y rendimiento en frijol bajo condiciones de secano. Agricultura Técnica en México, 34(1): 13-20.

Food and Agriculture Organization (FAO). 2017. Base de datos de producción agropecuaria FAOSTAT. Disponible en http://faostat3.fao.org/download/Q/QC/ E. 
Ministerio de Agricultura y Riego (MINAGRI). 2012. El Maíz Amiláceo. Principales aspectos de la cadena agroproductiva. Dirección General de Competitividad Agraria. Dirección de Información Agraria. Jr. Yauyos 258- Lima. 38 p. D i s p o i b l e e n : http://agroaldia.minagri.gob.pe/bibliote ca/download/pdf/agroeconomia/agroec onomiamaizamilaceo.pdf.

Monteith, J. L. 1977. Climate and the effi ciency of crop production in Britain. Phil Trans. Res. Soc. London Ser. B. 281: 277-329. D i s p n i b l e e n : http://rstb.royalsocietypublishing.org/c ontent/281/980/277

Paliwal, R. L. 2001. Introducción al maíz y su importancia. En: El maíz en los trópicos: mejoramiento y producción. Paliwal, R. L.; G. Granados; H. R. Laffite; A. D. Violic (Edes.). FAO, CIMMYT. Roma, 20001 . D i s p o n ib 1 e e n : https://curlacavunah.files.wordpress.co m/2010/04/el-maiz-en-los-tropicos.pdf.

Paliwal, R. L. and Sprague, E. W. 1981. Improving adaptation and yield dependability in maize in the developing world. Centro Internacional de Mejoramiento de Maíz y Trigo, CIMMYT Library. 20 p. Disponible en: http://libcatalog.cimmyt.org/Download/ cim/13186.pdf.

Quijano, C.; Aguirre, G. J.A. and Villarreal, F. E. 1995. Using crop simulation models to support agricultural research planning in Mexico. In: applaying crop models and decision support systems. University of Florida. International Fertilizer Development Center. $15 \mathrm{p}$.

Reta Sánchez, D. G.; Gaytán Mascorro, A.,
Carrillo Amaya, J. S. 2000. Rendimiento y componentes del rendimiento de maíz en respuesta a arreglos topológicos. Revista Fitotecnia Mexicana, 26(2): 7580 .

Ross, F. 2012. Densidad de plantas en maíz: Ajuste por ambiente. Cgacra Experimental Integrada MAA-INTA, Argentina. IAH 8: 11-14. Disponible en: http://www.ipni.net/publication/ialacs.nsf/0/7201946EC0B199E885257A C5006D65AA/\$FILE/11.dfp.

Sangoi, L. 2000. Understanding plan density effects on maize growth and development: an important issue to maximize grain yield. Ciência Rural, Santa Maria, 31(1):159-168.

Sangoi, L., Schmitt , A., Vieira, J., Picoli Jr., G. J., Souza, C. A., Trezzi Casa, R., Schenatto, D. E., Giordani, W., Majolo Boniatti, C., Cardoso Machado, G., e Horn, D. 2012. Variabilidade na distribuição espacial de plantas na linha e rendimento de grãos de milho. Revista Brasileira de Milho e Sorgo, 11(3): 268-277.

Sevilla P., R. 2008. El cultivo del maíz en el Perú. STC-CGIAR. 31 p. Disponible en: h t t p://www.psi.gob.pe/wp content/uploads/2016/03/biblioteca_exp osiciones_CULTIVO_DEL_-MAiZ.pdf

Tinoco Alfararo, C. A. 2006. Modelación del índice de área foliar y su relación con el rendimiento, en función del arreglo espacial de plantas en el cultivo de maíz. Trabajo de grado para optar al título de Doctor en Ciencias Agrícolas y Forestales. Universidad de Guadalajara. $62 \mathrm{p}$. D i s p o n i b 1 e e n : http://biblioteca.cucba.udg.mx:8080/xm lui/bitstream/handle/123456789/5171/ 
Tinoco_Alfaro_Carlos_Alberto.pdf?seq uence $=1$.

Tinoco Alfaro, C. A., Ramírez Fonseca, A., Villarreal Farías, E. y Ruiz Corral, A. 2008. Arreglo espacial de híbridos de maíz, índice de área foliar y rendimiento. Agricultura Técnica en México, 34(3): 271-278.

Van Heemst, H. D. J. and Van Keulen, H. 1986. Potencial crop production In: Van Keulen, H. and Wolf, J. (eds.), modelling of agricultural production: weather, soils and crops. Pudoc, Wagenigen; The Netherlands.

Versteeg, M. N. and Van Keulen, H. 1986. Potential crop production prediction by some simple calculation methods, as compared with computer simulations. Agricultural Systems, 19(4):249-272.

Videla Mensegue, H.; Canale, A. y Muñoz, S. 2014. Evaluación de diferentes densidades de plantas de maíz en siembras tempranas en ambientes contrastantes del Territorio Sudeste de Córdoba. Disponible en: https://www.researchgate.net/profile/H oracio_Videla_Mensegue/publication/2 70274811_Evaluacion_de_diferentes_d ensidades_de_plantas_de_maiz_en_sie mbras_tempranas_en_ambientes_contr astantes_del_Territorio_Sudeste_de_Co rdoba/links/56b094d008ae9c1968b78d 45 / Evaluacion-de-diferentes densidades-de-plantas-de-maiz-ensiembras-tempranas-en-ambientescontrastantes-del-Territorio-Sudeste-deCordoba.pdf.

\section{CORRESPONDENCIA}

Dr. Pedro García Mendoza

pejogam@gmail.com 